\title{
Advanced Cuckoo Search Algorithm for Optimization Problem
}

\author{
Nasif Shawkat \\ Ahsanullah University of \\ Science \& Technology \\ Dhaka, Bangladesh
}

\author{
Shariba Islam Tusiy \\ Ahsanullah University of \\ Science \& Technology \\ Dhaka, Bangladesh
}

\author{
Md. Arman Ahmed \\ Ahsanullah University of \\ Science \& Technology Dhaka, \\ Bangladesh
}

\begin{abstract}
For optimization tasks the most important and powerful algorithms are those which are inspired by the nature. The main goal of this work is the betterment of an existing algorithm called Cuckoo Search (CS) Algorithm. Cuckoo Search Algorithm (CS) is inspired from nature and related with Meta heuristic portion. This algorithm is based on random process and has behavior of some birds and fruit flies and some assumptions. Each assumption is highly observed to maintain their characteristics. This is a theoretical result research paper, of betterment for the Cuckoo Search (CS) Algorithm.
\end{abstract}

\section{General Terms}

Bio-Informatics, Meta heuristic.

\section{Keywords}

Meta heuristic; Bioinformatics; Nature Inspired Algorithms; Cuckoo Search (CS) algorithm; Improved Cuckoo Search (ICS) algorithm; Lévy flight.

\section{INTRODUCTION}

Beautiful nature is full of surprises and mysteries. People have learnt a lot from the Mother Nature and by analyzing symptoms people manage to reveal the mystery of nature. As time changes, humans also change their characteristics and their behavior to the nature [3][4]. The cuckoo search and the improved cuckoo search algorithm elevate the eco-life system in a new level. The Cuckoo Search and The Improved Cuckoo Search algorithm are extended to more complicated cases in which each nest has multiple eggs representing a set of solutions [5][6]. Within last few decades, dozens of metaheuristic algorithms are published and still been publishing. Among them Bat [7][8], Firefly [9][10], Flower Pollination [11], Artificial Bee Colony [1][12], Improved Artificial Bee colony [13], Ant Colony [14], Cuckoo search [15] are the highly recommended algorithms. Many meta-heuristic algorithms are inspired by the nature and they are becoming increasingly popular in modern world. This type of inspired algorithms is widely using in optimization problems like NPhard problems such as travelling sales man problem [19]. The algorithms which have mentioned above are upgrading day by day. Here it has been focused on the implementation and the operations of the iteration number, and the tested functions for both algorithms that mentioned above are same. For preparing this research, first of all, the data of mean, median and standard deviation for improved cuckoo search have been measured with the new analysis of best cuckoo search, which has presented by this paper and obtain success. Then, comparison makes them different from each other. By producing graphical outcome, it is observed that latest research of this paper which is best cuckoo search is good enough. "Cuckoo Search" (CS) \& its basic ideas are being described in section 2. Then in section 3, "Improved Cuckoo
Search" (ICS) is being described with its algorithm. After that in section 4 newly improved and advanced in cuckoo search algorithm is introduced. In section 5 the simulation \& analysis part is being described the new findings and graphs. Finally, in section 6 the total work is being summarized in short in the conclusion.

\section{CUCKOO SEARCH (CS)}

\subsection{Basic Ideas of Cuckoo Search}

Cuckoos maintain amazing intrusion reproductive strategy and it's known as brood parasitism. There are two types of brood parasitism: non-obligatory and obligatory [16][17]. In non-obligatory brood parasitism, cuckoos lay eggs in the nest of same species and in their own nests as well like Bank Swallows, African Weavers [18]. On the other hand in obligatory brood parasitism cuckoos lay eggs in the nest of hetero-specifies, and do not require building the nest of their own and incubate the eggs like Brown- Headed Cowbirds and European Cuckoos. It has been reported that obligatory brood parasitism followed by $1 \%$ of all bird species. For example, all African Honey Guides, almost half of the species of cuckoos, the Black Headed Duck in South America, Shiny Cowbirds, Screaming Cowbirds, Bronze Cowbirds and Giant Cowbirds. Some female cuckoo like Guira and Ani can copy the patterns and colors of few chosen host species. This imitate power is used to increase the hatching probability among them, which brings their next generation. A cuckoo maintains an amazing timing of laying eggs. The parasitic cuckoos used to choose a nest where the host birds lay their own eggs. It takes less time to hatch cuckoo's egg than the host bird's eggs. After hatching the first egg, their first instinct action is to throw out the hosts' eggs or to propel the eggs out of the nest to ensure the food from the host bird [2].

\subsection{Basic Points of Cuckoo Search}

Each Cuckoo's egg, which is placed in a nest, illustrates a new solution. The aim of Improved Cuckoo Search is to serve the new and potentially better solutions to replace the previous solutions in the Cuckoo Search. The algorithm can be extended to more complicated cases in which each nest has multiple eggs that represent a set of solutions. The CS is based on three idealized rules that are given bellow:

1. Each cuckoo lays one egg at a certain time, and dumps it in a nest which is randomly chosen [18].

2. The best nests provide high quality of eggs (solutions) that will carry over to the next generations [18].

3. A host bird can discover an alien egg from his nest with probability of $\mathrm{Pa} \in[0,1]$. In this case, the host bird can either throw the egg away or abandon or can completely build a new nest in a new location [18]. 


\subsection{Lévy Flights}

Many animals and insects in nature follow the properties of Lévy Flight. After the French mathematician Paul Pierre Lévy, the name Lévy Flight came. The term Lévy Flight was invented by Benoit Mandelbrot who used specific definition of the distribution of the step sizes. After some time Lévy Flight term started using to refer discrete grid rather than continuous space. Lévy Flight is a Markov Process. Exponential property of Lévy Flight gives it a scale invariant property and they are used to model data for displaying clusters. Reynolds and Frye in recent studies showed that fruit flies cover the skies by using numerous series of straight flight paths followed by a sudden right angle turn and it is a Lévyflight-style intermittent scale free. The term Lévy Flight can be thought of as a random walk where the step size has a Lévy tailed probability distribution. The flight behavior of many animals and insects demonstrates the typical characteristics of Lévy flights. A Lévy flight is a random walk in which the step-lengths are distributed according to a heavy probability distribution. After a large number of steps, the distance from the origin of the random walk tends to a stable distribution [2][19][20].

\section{IMPROVED CUCKOO SEARCH (ICS)}

\subsection{Characteristics of Improved Cuckoo Search}

The parameters $\mathrm{Pa}, \lambda$ and $\alpha$ introduced in the CS, help the algorithm to find globally and locally improved solutions, respectively. The parameters $\mathrm{Pa}$ and $\alpha$ is very important parameters in fine-tuning of solution vectors, and can be potentially used to adjust the convergence rate of the algorithm. The traditional CS algorithm uses a fixed value for both $\mathrm{Pa}$ and $\alpha$. The key difference between ICS and CS is the way of adjusting $\mathrm{Pa}$ and $\alpha$. To improve the performance of CS algorithm and eliminate the drawbacks lies with fixed values of $\mathrm{Pa}$ and $\alpha$, the ICS algorithm uses variables $\mathrm{Pa}$ and $\alpha$. The values of $\mathrm{Pa}$ and $\alpha$ dynamically change with the number of generations and have been expressed in equations 1-3, where $\mathrm{NI}$ and gn are the number of total iterations and the current iteration respectively.

$$
\begin{gathered}
P_{a}\left(g_{n}\right)=P_{a \max }-\frac{g_{n}}{N I}\left(P_{a \max }-P_{a \min }\right) \\
\alpha\left(g_{n}\right)=\alpha_{\max } \exp \left(\text { c. } g_{n}\right) \\
c=\frac{1}{N I} L_{n}\left(\frac{\alpha_{\min }}{\alpha_{\max }}\right)
\end{gathered}
$$

\subsection{Algorithm of ICS}

Begin

Objective function $\mathrm{f}(\mathrm{x}), \mathrm{x}=(\mathrm{x} 1, \ldots, \mathrm{xd}) \mathrm{T}$;

Initial a population of $\mathrm{n}$ host nests $\mathrm{xi}(\mathrm{i}=1,2, \ldots, \mathrm{n})$;

While ( $<$ MaxGeneration) or (stop criterion)

Get a cuckoo (say i) randomly by Lévy flights;

Evaluate its quality/fitness Fi;

Choose a nest among n (say j) randomly;

if $(\mathrm{Fi}>\mathrm{Fj})$ Replace $\mathrm{j}$ by the new solution;

endif

A fraction $(\mathrm{Pa})$ of worse nests are abandon and

New ones are built.
Keep the best solutions (or nests with quality solutions);

Rank the solutions and find the current best;

End while

Post-process results and visualization;

End

When generating new solutions $\mathrm{Xi}(\mathrm{t}+1)$ for the ith cuckoo, the following Lévy flight is performed

$$
X_{i}(t+1)=X_{i}(t)+\alpha \oplus \text { Lévy }(\lambda)
$$

Where $\alpha>0$ is the step size, which should be related to the scale of the problem of interest. The product $\oplus$ means an entry-wise multiplications. According to Yang's research work, it has considered that a Lévy flight in which the steplengths are distributed according to the following probability distribution

$$
\text { Lévy } \mathrm{u}=\mathrm{t}-\lambda, 1<\lambda \leq 3
$$

This has an infinite variance. Above here, the consecutive steps of a cuckoo essentially form a random walk process that obeys a power law step length distribution with a heavy tail.

It is worth pointing out that, in the real world, if a cuckoo's egg is very similar to a host's egg, then this cuckoo's egg is less likely to be discovered, thus the fittest should be related to the difference in solutions. Therefore, it is a good idea to do a random walk in a biased way with some random step sizes [17][18].

\section{ADVANCED CUCKOO SEARCH (ACS)}

The basic concepts of cuckoo search by Lévy flight optimization are being followed in this algorithm. The newly improved best cuckoo search has some changes in the raw code to get more effective result, and the simulations are good for this change. By improving some parameters and some conditions, this research brought better results than before. In ACS, there has some changes from basic cuckoo search algorithm. Although it's based on Lévy flights optimization algorithm it have some scope to get better result than the previous algorithm.

Begin

Objective function $\mathrm{f}(\mathrm{x}), \mathrm{x}=(\mathrm{x} 1, \ldots, \mathrm{xd}) \mathrm{T}$

Generate initial population of $n$ host nests $x i(i=1,2, \ldots, n)$

While $(\mathrm{t}<$ MaxGeneration) or (stop criterion)

Get a cuckoo randomly by Lévy flights

Evaluate its quality/fitness Fi;

Choose a nest among $n(s a y, j)$ randomly If $(\mathrm{Fi}>\mathrm{Fj})$,

Replace $\mathrm{j}$ by the new solution;

End

A fraction $(\mathrm{Pa})$ of worse nests are abandon and

New ones are built;

Keep the best solutions (or nests with quality solutions);

Rank the solutions and find the current best;

End while 
Post-process results and visualization;

End

For ACS, the discovery rate of eggs/solutions are set as $\mathrm{pa}=.25$ and iteration number varies for random search and the values are taken as $1000,2000,3000$. The number of dimensions are also varies and the values are taken as $n=10$, 15, 25. Finding the fitness of cuckoo search is the best objective. If the cuckoo's new fitness is greater than the cuckoo's previous fitness then there is huge possibility to find the best nest for cuckoo in the new search area from where cuckoo can find their nearest nest. And this decision gives ACS to find a good result for cuckoo search which is better than ICS.

\section{SIMULATION AND ANALYSIS}

\subsection{Comparison studies}

The simulation parts are divided in three sections as the calculations are separated respect to the number of dimension. For $\mathrm{n}=10$ dimension and for 1000 iteration, the Easom function gives the best value among all 15 functions rather than ICS. And for all functions our ACS gives the best result than ICS. And for Goldstein function, it gives best result for ICS rather than ACS. So the Goldstein function gives worst result for ACS. For $\mathrm{n}=15$ dimension and for 2000 iteration, the Quartic function gives the best value among all 15 functions rather than ICS. And for all functions our ACS gives the best result than ICS. And for Ackley function, it gives best result for ICS rather than ACS. So the Ackley function gives worst result for ACS. For $\mathrm{n}=25$ dimension and for 3000 iteration, the Goldstein function gives the best value among all 15 functions rather than ICS. And for all functions our ACS gives the best result than ICS. And for Rosenbrock function, it gives best result for ICS rather than ACS. So the Rosenbrock function gives worst result for ACS.

All the dimensions are calculated for 15 functions which are given with their parameters. Some of them are given in next section.

\subsection{Test Functions}

There are various benchmark test functions to verify and get the performance of optimization algorithms. Almost every optimization algorithms must be tested and verified against these benchmark functions. In this algorithm (ACS), few test functions have been utilized for simulations

a) Sphere Function

$$
\min f_{1}=\sum_{i=1}^{N} x_{i}^{2}
$$

b) Rosenbrock Function

$$
\min f_{2}=\sum_{i=1}^{N-1}\left(100\left(x_{i-1}-x_{i}^{2}\right)^{2}+\left(x_{i}-1\right)^{2}\right)
$$

c) Kowalik Function

$$
f_{3}(x)=\sum_{t-1}^{11}\left[a_{t}-\frac{x_{1}\left(b_{t}^{2}+b_{t} x_{2}\right)}{b_{t}^{2}+b_{t} x_{3}+x_{4}}\right]^{2}
$$

d) Ranstrigrin Function

$$
\min f_{4}=\sum_{i=1}^{N}\left(x_{i}^{2}-10 \cos \left(2 \pi x_{i}\right)+10\right)
$$

e) Griewank Function

$$
\min f_{5}=\frac{1}{4000} \sum_{i=1}^{N} x_{i}^{2}-\prod_{i=1}^{N} \cos \left(\frac{x_{i}}{\sqrt{i}}\right)+1
$$

f) Ackley's Function

$$
\begin{array}{r}
\min f_{6}=20+e-20 \exp \left(-0.2 \sqrt{\frac{\sum_{i=1}^{N} x_{i}^{2}}{N}}\right) \\
-\exp \left(\frac{\sum_{i=1}^{N} \cos \left(2 \pi x_{i}\right)}{N}\right)
\end{array}
$$

g) Zakharov Function

$$
f_{7}(\vec{x})=\sum_{i=1}^{D} x_{i}^{2}+\left(\frac{1}{2} \sum_{i=1}^{D} i x_{i}\right)^{2}+\left(\frac{1}{2} \sum_{i=1}^{D} i x_{i}\right)^{4}
$$

h) Schwefel Function

$$
\min f_{8}=418.9829 N-\sum_{i=1}^{N}\left(x_{i} \sin \left(\sqrt{\left|x_{i}\right|}\right)\right)
$$

i) Easom Function

$$
f_{9}(x, y)=-\cos (x) \cos (y) \exp \left[-(x-\pi)^{2}-(y-\pi)^{2}\right]
$$

j) Michalewicz Function

$$
f(x)=-\sum_{i=1}^{d} \sin \left(x_{i}\right)\left[\sin \left(\frac{i x_{i}^{2}}{\pi}\right)\right]^{2 m}
$$

An algorithm is specified as a good balance between ICS

\begin{tabular}{|c|c|c|c|c|c|c|}
\hline Function & $\begin{array}{c}\text { Algori } \\
\text { thm }\end{array}$ & $\begin{array}{l}\text { Iterati } \\
\text { on }\end{array}$ & $\begin{array}{l}\text { Di } \\
\text { m }\end{array}$ & Mean & $\begin{array}{l}\text { Medi } \\
\text { an }\end{array}$ & SD \\
\hline \multirow{6}{*}{ Sphere } & ACS & 1000 & \multirow{2}{*}{10} & $\begin{array}{c}0.449 \\
141\end{array}$ & $\begin{array}{c}0.85 \\
5042\end{array}$ & $\begin{array}{c}1.847 \\
431\end{array}$ \\
\hline & ICS & 1000 & & $\begin{array}{c}4.705 \\
451\end{array}$ & $\begin{array}{l}0.98 \\
6727\end{array}$ & $\begin{array}{c}5.872 \\
553\end{array}$ \\
\hline & ACS & 2000 & \multirow{2}{*}{15} & $\begin{array}{c}1.625 \\
41\end{array}$ & $\begin{array}{l}1.56 \\
715\end{array}$ & $\begin{array}{c}1.811 \\
411\end{array}$ \\
\hline & ICS & 2000 & & $\begin{array}{c}1.685 \\
181\end{array}$ & $\begin{array}{c}1.28 \\
3744\end{array}$ & $\begin{array}{c}1.469 \\
723\end{array}$ \\
\hline & ACS & 3000 & \multirow{2}{*}{25} & $\begin{array}{c}0.765 \\
654\end{array}$ & $\begin{array}{l}0.14 \\
544\end{array}$ & $\begin{array}{c}1.791 \\
581\end{array}$ \\
\hline & ICS & 3000 & & $\begin{array}{c}3.664 \\
342\end{array}$ & $\begin{array}{l}1.14 \\
895\end{array}$ & $\begin{array}{c}4.216 \\
293\end{array}$ \\
\hline Step & ACS & 1000 & 10 & $\begin{array}{c}0.449 \\
141\end{array}$ & $\begin{array}{l}0.85 \\
504\end{array}$ & $\begin{array}{c}1.847 \\
431\end{array}$ \\
\hline
\end{tabular}
algorithm and the newly approached algorithm. It gives the best result than ICS which is the last modified algorithm of cuckoo search. The calculations of this and the comparison tables are given to have a clear look of this search. After this new ACS, there are few new scopes of research have come out with more complex parameters.

Table 1: Comparison between ACS \& ICS on 15 standard benchmark functions. Both algorithms are run 45 different times on each of the functions. The best result for each function with each dimension is marked as bold 


\begin{tabular}{|c|c|c|c|c|c|c|}
\hline & ICS & 1000 & & $\begin{array}{c}2.850 \\
699\end{array}$ & $\begin{array}{l}0.87 \\
805\end{array}$ & $\begin{array}{c}3.136 \\
773\end{array}$ \\
\hline & ACS & 2000 & \multirow{2}{*}{15} & $\begin{array}{c}0.004 \\
878\end{array}$ & $\begin{array}{c}- \\
0.50 \\
369\end{array}$ & $\begin{array}{c}2.169 \\
358\end{array}$ \\
\hline & ICS & 2000 & & $\begin{array}{c}5.187 \\
205\end{array}$ & $\begin{array}{c}0.57 \\
525\end{array}$ & $\begin{array}{c}6.857 \\
659\end{array}$ \\
\hline & ACS & 3000 & \multirow{2}{*}{25} & $\begin{array}{c}1.837 \\
399\end{array}$ & $\begin{array}{l}2.34 \\
712\end{array}$ & $\begin{array}{c}2.030 \\
147\end{array}$ \\
\hline & ICS & 3000 & & $\begin{array}{c}2.380 \\
568\end{array}$ & $\begin{array}{c}0.95 \\
418\end{array}$ & $\begin{array}{c}2.417 \\
289\end{array}$ \\
\hline \multirow{6}{*}{ Zakharov } & ACS & 1000 & \multirow{2}{*}{10} & $\begin{array}{c}1.161 \\
907\end{array}$ & $\begin{array}{l}1.09 \\
051\end{array}$ & $\begin{array}{c}1.731 \\
236\end{array}$ \\
\hline & ICS & 1000 & & $\begin{array}{c}5.263 \\
648\end{array}$ & $\begin{array}{l}1.00 \\
930\end{array}$ & $\begin{array}{c}6.196 \\
252\end{array}$ \\
\hline & ACS & 2000 & \multirow{2}{*}{15} & $\begin{array}{c}0.182 \\
078\end{array}$ & $\begin{array}{l}0.83 \\
037\end{array}$ & $\begin{array}{c}1.983 \\
451\end{array}$ \\
\hline & ICS & 2000 & & $\begin{array}{c}3.691 \\
843\end{array}$ & $\begin{array}{l}1.15 \\
223\end{array}$ & $\begin{array}{c}4.383 \\
291\end{array}$ \\
\hline & ACS & 3000 & \multirow{2}{*}{25} & $\begin{array}{c}0.330 \\
762\end{array}$ & $\begin{array}{l}0.80 \\
780\end{array}$ & $\begin{array}{c}1.937 \\
459\end{array}$ \\
\hline & ICS & 3000 & & $\begin{array}{c}4.325 \\
622\end{array}$ & $\begin{array}{c}0.66 \\
793\end{array}$ & $\begin{array}{c}5.563 \\
92\end{array}$ \\
\hline \multirow{6}{*}{ Griewangk } & ACS & 1000 & \multirow{2}{*}{10} & $\begin{array}{c}1.592 \\
954\end{array}$ & $\begin{array}{l}2.16 \\
436\end{array}$ & $\begin{array}{c}2.272 \\
2\end{array}$ \\
\hline & ICS & 1000 & & $\begin{array}{c}4.595 \\
908\end{array}$ & $\begin{array}{l}0.48 \\
267\end{array}$ & $\begin{array}{c}5.897 \\
144\end{array}$ \\
\hline & ACS & 2000 & & $\begin{array}{c}0.907 \\
428\end{array}$ & $\begin{array}{l}0.81 \\
328\end{array}$ & $\begin{array}{c}2.304 \\
591\end{array}$ \\
\hline & ICS & 2000 & IJ & $\begin{array}{c}5.057 \\
924\end{array}$ & $\begin{array}{l}0.84 \\
877\end{array}$ & $\begin{array}{c}6.421 \\
581\end{array}$ \\
\hline & ACS & 3000 & 25 & $\begin{array}{c}0.451 \\
748\end{array}$ & $\begin{array}{c}- \\
0.12 \\
132\end{array}$ & $\begin{array}{c}2.079 \\
286\end{array}$ \\
\hline & ICS & 3000 & & $\begin{array}{c}3.670 \\
911\end{array}$ & $\begin{array}{l}1.08 \\
075\end{array}$ & $\begin{array}{c}4.363 \\
651\end{array}$ \\
\hline \multirow{6}{*}{ Rastrigin } & ACS & 1000 & \multirow{2}{*}{10} & $\begin{array}{c}1.194 \\
819\end{array}$ & $\begin{array}{l}0.96 \\
729\end{array}$ & $\begin{array}{c}2.253 \\
5\end{array}$ \\
\hline & ICS & 1000 & & $\begin{array}{c}3.939 \\
145\end{array}$ & $\begin{array}{c}0.42 \\
132\end{array}$ & $\begin{array}{c}5.157 \\
28\end{array}$ \\
\hline & ACS & 2000 & \multirow{2}{*}{15} & $\begin{array}{c}0.860 \\
391\end{array}$ & $\begin{array}{l}1.15 \\
331\end{array}$ & $\begin{array}{c}1.685 \\
941\end{array}$ \\
\hline & ICS & 2000 & & $\begin{array}{c}2.633 \\
448\end{array}$ & $\begin{array}{c}0.81 \\
493\end{array}$ & $\begin{array}{c}2.878 \\
158\end{array}$ \\
\hline & ACS & 3000 & \multirow{2}{*}{25} & $\begin{array}{c}0.438 \\
344\end{array}$ & $\begin{array}{l}1.34 \\
461\end{array}$ & $\begin{array}{c}2.124 \\
764\end{array}$ \\
\hline & ICS & 3000 & & $\begin{array}{c}4.466 \\
253\end{array}$ & $\begin{array}{l}1.12 \\
937\end{array}$ & $\begin{array}{c}5.406 \\
639\end{array}$ \\
\hline \multirow{2}{*}{$\begin{array}{c}\text { Rosenbroc } \\
\mathbf{k}\end{array}$} & ACS & 1000 & \multirow[t]{2}{*}{10} & $\begin{array}{c}0.789 \\
953\end{array}$ & $\begin{array}{l}0.19 \\
623\end{array}$ & $\begin{array}{c}1.814 \\
792\end{array}$ \\
\hline & ICS & 1000 & & 2.969 & 0.90 & 3.340 \\
\hline
\end{tabular}

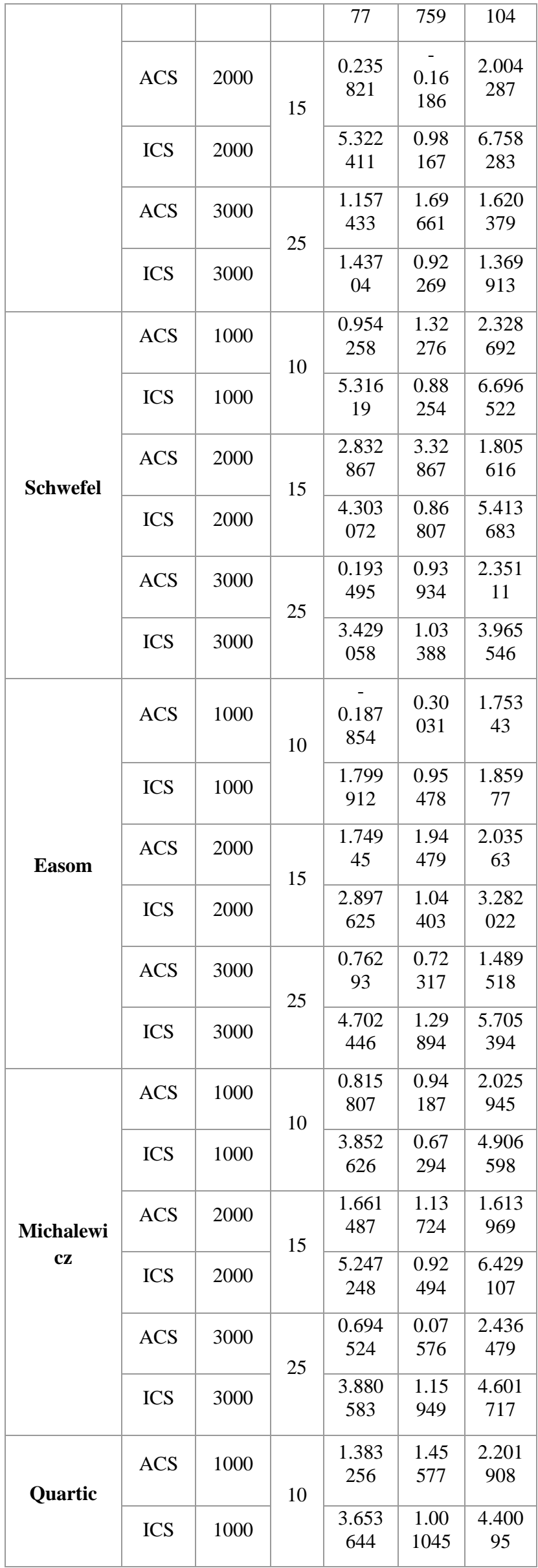




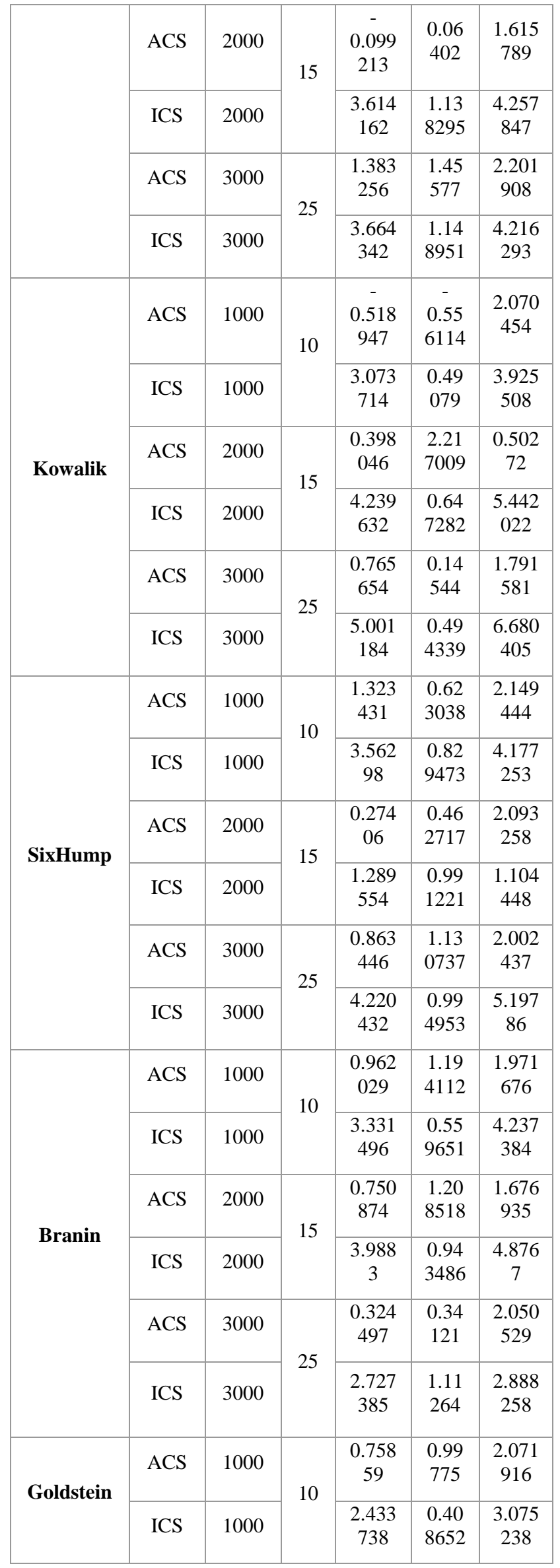

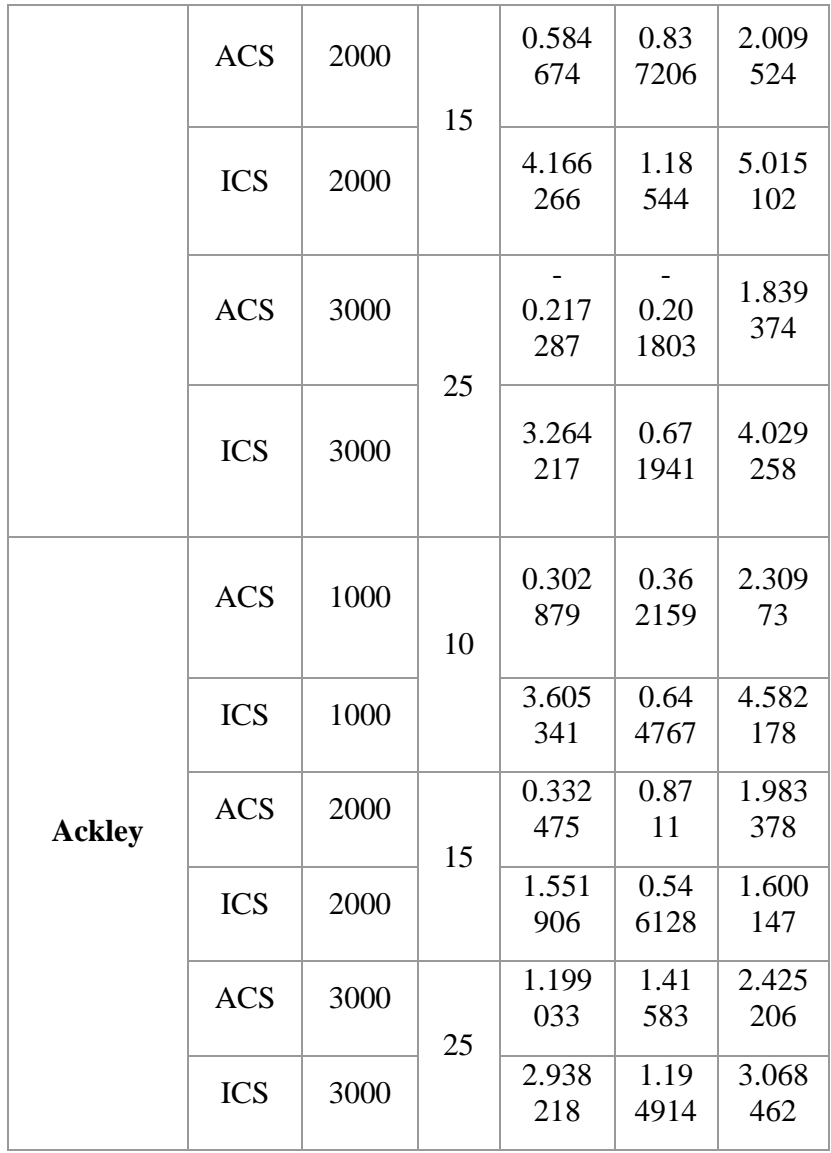

\subsection{Graphs}

\subsubsection{Parameter settings}

In this paper, we have tested those algorithms with 1000 , 2000, 3000 independent iterations, on each of the test functions. The Dimension for each function for each algorithm is set for $\mathrm{D}=10,15,25$ respectively. For ACS we use $\mathrm{Pa}, \alpha, \lambda$ to improve the result for ACS globally and locally. Here in ACS we used Discovery rate of alien eggs or solutions, $\mathrm{Pa}=0.25$. We have found the best, worst, mean, median and Standard deviation value for both algorithm. On the basis of the result of finding best and new place as well as nest or colony we simulated 3D surface and mesh for Easom function on ACS. Here we have plotted these graphs on the basis of new nests of cuckoo. We have showed 3D surface, 3D mesh. We used matlab R2013a version for simulation with 4 th generation Intel i5 processor $2.7 \mathrm{GZ}$ with $4 \mathrm{~GB}$ RAM of PC. 


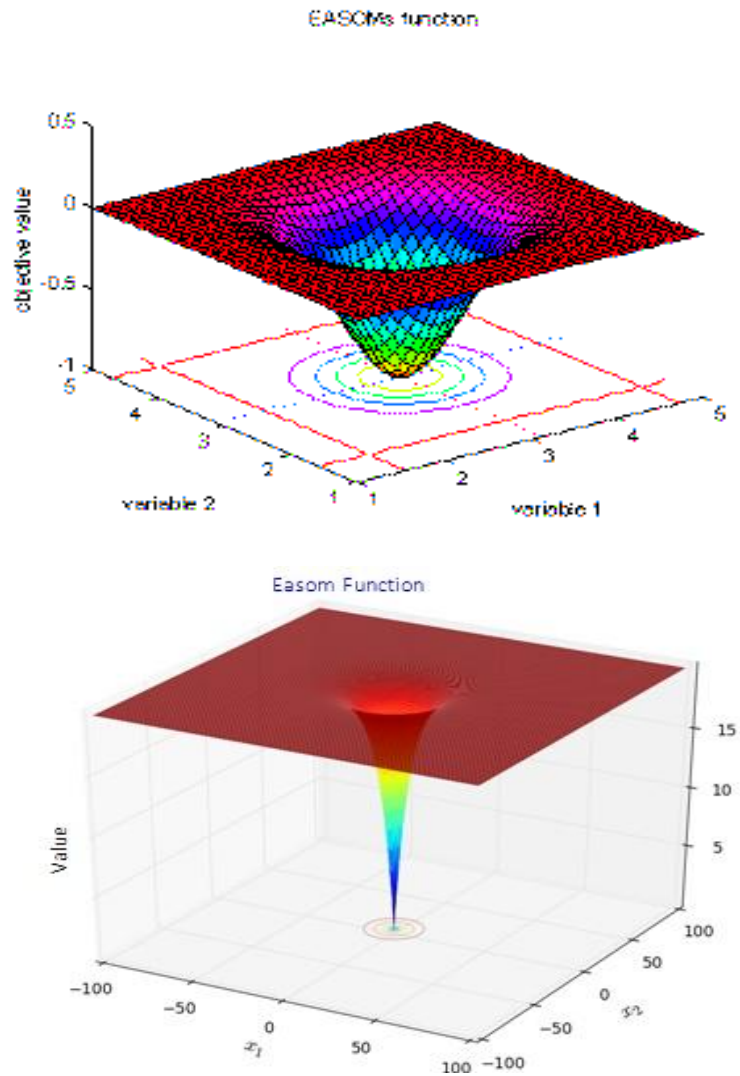

Fig: 3D surface plots (2 view) Easom function that best for ACS

\section{CONCLUSION}

This paper mainly focus on the Lévy flight behavior base algorithm Improved Cuckoo Search and Cuckoo Search. The result is much better than the Improved Cuckoo Search which has shown with necessary diagram above. Different types of graphs and values for different functions are showing the differences. The algorithm of cuckoo search has been modified and tested for different dimensions and iterations. Fifteen different types of functions for different dimensions and iterations have been used to check the result and finally the new result is much better than the improved cuckoo search. So it can be conclude that, proposed result is much better than the improved cuckoo search.

\section{REFERENCES}

[1] D. Karaboga, "An Idea Based On Honey Bee Swarm for Numerical Optimization," Technical Report-TR06, Erciyes University, Engineering Faculty, Computer Engineering Department, 2005.

[2] S. I. Tusiy, N. Shawkat, M. A. Ahmed, B. Panday and N. Sakib, "Comparative Analysis of Improved Cuckoo Search(ICS) Algorithm and Artificial Bee Colony (ABC) Algorithm on Continuous Optimization Problems," International Journal of Advanced Research in Artificial Intelligence(IJARAI), vol. 4, no. 2, pp. 14-19, 2015.

[3] X. S. Yang, "Nature-Inspired Metaheuristic Algorithms," LuniverPress,UK, 2008.

[4] IJAIA, "International Journal of Artificial Intelligence \& Applications," vol. 2, July 2011.
[5] M. Gendreau, "Handbook of Metaheuristics," in An introduction to tabu search, Kluwer Academic Publishers, 2003, p. 37-54.

[6] X. Yang and S. Deb, "Engineering Optimisation by Cuckoo Search," in Int. J. Mathematical Modelling and Numerical Optimisation, 2010, p. 330-343.

[7] X.-S. Yang, "Bat algorithm: literature review and applications," Int. J. Bio-Inspired Computation, vol. 5, p. $141-149,2013$.

[8] I. J. Fister, D. Fister and X.-S. Yang, "A hybrid bat algorithm," in Elek-trotehniskivestnik, press, 2013.

[9] X.-S. Yang and s. H. Xing, "Firefly Algorithm: Recent Advances and Applications," Int. J. Swarm Intelligence, vol. 1, p. 36-50, 2013.

[10] X.-S. Yang, "Firefly algorithms for multimodal optimization," Stochastic Algorithms: Foundations and Applications, SAGA 2009, Lecture Notes in Computer Sciences, vol. 5792, pp. 169-178, 2009.

[11] X.-S. Yang, "Flower pollination algorithm for global optimiza-tion," Unconventional Computation and Natural Computation 2012,Lecture Notes in Computer Science, vol. 7445, pp. 240-249, 2012.

[12] D. Karaboga, "An Idea Based on Honey Bee Swarm for Numerical Optimization.Technical Report-TR06," in Erciyes University, Computer Engineering Department, 2005.

[13] M. Kiran and A. Babalik, "Improved Artificial Bee Colony Algorithm for Continuous Optimization Problems," Journal of Computer and Communications, vol. 2, pp. 108-116, 2014.

[14] A. Farzindar and V. (. Keselj, Canadian AI 2010, LNAI 6085,2010

[15] I. J. Fister, X. Yang, D. Fister and I. Fister, "Cuckoo search: A brief literature review," Cuckoo Search and Firefly Algorithm: Theory and Applications, Studies in Computational Intelligence, vol. 516, pp. 49-62, 2014.

[16] X.-S. Yang, Optimization Problem, Department of Engineering,University of Cambridge, Trumpinton Street, Cambridge CB2 1PZ, UK.

[17] M. Gendreau, "An introduction to tabu search," in Handbook of Metaheuristics, Kluwer Academic Publishers, 2003, p. 37-54.

[18] E. Valian, S. Mohanna and S. Tavakoli, "Improved Cuckoo Search Algorithm for Global Optimization," University of Sistan and Baluchestan, Dec. 2011.

[19] X.-S. Yang, Cuckoo Search via L'evy Flights, Department of Engineering,University of Cambridge, Trumpinton Street, Cambridge CB2 1PZ, UK. Clifford T. Brown, "Lévy Flights in DobeJu/'hoansi Foraging Patterns," 777 Glades Road, Boca Raton, FL 33431, USA, Department of Anthropology, Florida Atlantic University , 6 December 2006, p. 129-138. 